\title{
Mechanism of transdermal permeation promotion of lipophilic drugs by ethosomes
}

\author{
This article was published in the following Dove Press journal: \\ International Journal of Nanomedicine \\ 26 April 2017 \\ Number of times this article has been viewed
}

\author{
Li Yangl,* \\ Lifang $\mathrm{Wu}^{1}{ }^{1, *}$ \\ Dongze $\mathrm{Wu}^{2}$ \\ Deshun $\mathrm{Shi}^{3}$ \\ Tai Wang ${ }^{4}$ \\ Xiaoliang $\mathrm{Zhu}^{4}$ \\ 'Department of Dermatology, \\ Nanfang Hospital, Southern Medical \\ University, Guangzhou, ${ }^{2}$ Department \\ of Medicine and Therapeutics, The \\ Prince of Wales Hospital, The Chinese \\ University of Hong Kong, Hong Kong, \\ ${ }^{3}$ Department of Dermatology, The \\ First People's Hospital of Foshan, \\ Foshan, ${ }^{4}$ Department of Dermatology, \\ Nanfang Hospital, Southern Medical \\ University, Guangzhou, People's \\ Republic of China \\ *These authors contributed equally \\ to this work
}

\begin{abstract}
Ethosomes can promote the penetration of lipophilic drugs into the skin, but the underlying mechanism is still unknown. The purpose of this study was to investigate the mechanism of transdermal permeation promotion of lipophilic drugs by ethosomes. The formulation of ethosomes was optimized using the Box-Behnken experimental design, in which Rhodamine B and 1-palmitoyl-2-\{12-[(7-nitro-2-1,3-benzoxadiazol-4-yl)amino]dodecanoyl\}-sn-glycero-3phosphocholine were used to simulate a model lipophilic drug and act as a fluorescent tracer of ethosomal phospholipids, respectively. Liposomes with the same phospholipid concentration and a hydroethanolic solution with the same ethanol concentration were also prepared as controls. The percutaneous progression of the above fluorescent preparations was observed by confocal laser scanning microscopy, and the fluorescence intensity of the images was analyzed. The optimized ethosome formulation consisted of $2.45 \%$ yolk phospholipids, $30 \%$ ethanol, and $67.55 \%$ distilled water. The percutaneous permeation of Rhodamine B in the optimized ethosomes was superior to that in hydroethanolic solution $(P<0.05)$ and liposomes $(P<0.05)$. The ethosomes could penetrate the skin via the percutaneous pathway of the hair follicle and stratum corneum, while during the process of penetration, the vesicles were broken and the phospholipids were retained in the upper epidermis, with the test compounds penetrating gradually. The superior percutaneous penetration of ethosomes was linked to the synergistic effects of their ingredients. The percutaneous pathways of ethosomes included open hair follicles and stratum corneum pathways. In addition, the vesicles might break up during percutaneous penetration in the superficial layer of the skin, allowing the test compounds to keep permeating into the deeper layer alone, while the phospholipid was retained in the upper epidermis.
\end{abstract}

Keywords: ethosomes, mechanism, percutaneous, confocal laser scanning microscopy, CLSM

\section{Introduction}

Many lipophilic drugs, including fusidic acid, ${ }^{1}$ bifonazole, ${ }^{2}$ valaciclovir, ${ }^{3}$ corticosteroid, ${ }^{4}$ and tacrolimus, ${ }^{5}$ are used in the treatment of various skin diseases. Formulating these drugs as creams or emulsions could have advantages over other methods of administration, including avoidance of first-pass metabolism, a lower frequency of administration, and improved patient compliance. However, topical skin formulations remain ineffective due to a low percutaneous penetration through the stratum corneum. There are increasing efforts to overcome the powerful barrier of the stratum corneum. Such approaches include the use of iontophoresis, ${ }^{6}$ electroporation, ${ }^{7}$ microneedles, ${ }^{8}$ sonophoresis, ${ }^{9}$ surfactants, ${ }^{10}$ and liposomal-based nanoparticles $;{ }^{11}$ however, the deficiencies associated with these methods, such as complicated processing, skin injury, patient compliance, poor stability, and high cost, have significantly limited their widespread applications in clinical practice.
Correspondence: Xiaoliang Zhu Department of Dermatology, Nanfang Hospital, Southern Medical University, 1838 North Guangzhou Avenue,

Guangzhou, Guangdong 510515, People's

Republic of China

Tel +862062786258

Fax +862061641049

Emailnfnpfk@I63.com
International Journal of Nanomedicine 2017:12 3357-3364

3357

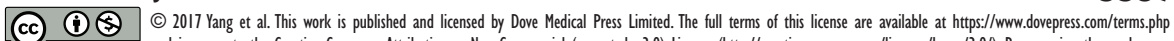

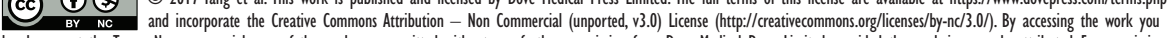
hereby accept the Terms. Non-commercial uses of the work are permitted without any further permission from Dove Medical Press Limited, provided the work is properly attributed. For permision for commercial use of this work, please see paragraphs 4.2 and 5 of our Terms (https://www.dovepress.com/terms.php). 
As a novel lipid vehicle, ethosomes have shown superb effectiveness in percutaneous drug delivery. In addition, they have superior pharmaceutical properties, including stability at room temperature, high entrapment efficiency, and improved compatibility with the stratum corneum, thus promoting the effective penetration of lipophilic drugs into the skin. ${ }^{12-14}$ In our preliminary study, we have proven that ethosomes can promote transdermal permeation of lipophilic drugs, such as lidocaine, with a shorter onset time and a longer duration in vivo than lidocaine-based liposomes or lidocaine delivered in a hydroethanolic solution. ${ }^{15}$ Subsequently, ethosomes have been used in photodynamic therapy, ${ }^{16,17}$ cosmeceuticals, ${ }^{18}$ and a vaccine. ${ }^{19}$

However, the mechanisms by which ethosomes promote skin penetration of drugs remain elusive. ${ }^{20,21}$ Therefore, the following three key questions are critical: Which key ingredient(s) in ethosomes is responsible for their excellent properties in promoting percutaneous penetration? Can ethosomes penetrate into the skin with an intact vesicle structure? What is the detailed mechanism by which ethosomes loaded with lipophilic drugs penetrate into the skin?

In this study, we adopted Rhodamine B to simulate a model lipophilic drug and 1-palmitoyl-2-\{12-[(7-nitro-21,3-benzoxadiazol-4-yl)amino]dodecanoyl \}-sn-glycero-3phosphocholine (NBD-PC) as a tracer of phospholipids to prepare fluorescent ethosomes. The aims of this study were to evaluate the percutaneous efficiency of model drug-loaded ethosomes as well as to determine the mechanisms related to lipophilic drug penetration into the skin.

\section{Materials and methods}

\section{Chemicals and reagents}

The compound NBD-PC was purchased from Avanti Polar Lipids (Alabaster, AL, USA). Rhodamine B, hexyl ester, perchlorate was obtained from AAT Bioquest (Sunnyvale, CA, USA). High-purity yolk phospholipids (98\%) were provided by Nippon Fine Chemical Co., Ltd. (Tokyo, Japan). Chloroform and $100 \%$ ethanol were purchased from GuangHua Company (Guangzhou, People's Republic of China). All other reagents and solvents used in this study were of analytical grade and were obtained from local companies.

\section{Animals}

Specific pathogen-free male Sprague Dawley rats, weighing 220-250 $\mathrm{g}$ and aged 7-8 weeks, were obtained from the Laboratory Animal Center, Southern Medical University, Guangzhou, People's Republic of China. The animals were housed in a room under a controlled temperature $\left(25^{\circ} \mathrm{C} \pm 2^{\circ} \mathrm{C}\right)$ and humidity $(50 \% \pm 2 \%)$, with free access to standard laboratory chow and tap water in an iron cage. The animal use and care protocols were reviewed and approved by the Institutional Animal Care and Use Committee of Southern Medical University. All procedures of the animal experiments adhered to the Principles of Laboratory Animal Care guidelines of the Laboratory Animal Center, Southern Medical University, following the principles of the Declaration of Helsinki.

\section{Experimental design to optimize the ethosome formulation}

Using the vesicle size as a dependent variable, we optimized the following independent variables: yolk phospholipid content $(1 \%-5 \%, \mathrm{w} / \mathrm{v})$, ethanol content $(20 \%-30 \%, \mathrm{v} / \mathrm{v})$, and ultrasonic treatment time (6-8 $\mathrm{min})$, with each being set at low (-1), medium (0), and high (+1) levels. We employed a Box-Behnken experimental design, ${ }^{22}$ with three factors, three levels, and 17 runs in the optimization study, using DesignExpert.V8.0.6 (Stat-Ease, Inc., Minneapolis, MN, USA). Three-dimensional response surface plots were generated for the main effects and interactive responses to obtain the optimized vesicle size.

\section{Preparations of ethosomes and other formulations}

To optimize the composition of the ethosomes, various formulations of ethosomes based on the above Box-Behnken design were prepared by applying the sonication method reported previously. ${ }^{23}$ Yolk phospholipid was dissolved along with Rhodamine B $(0.02 \%, \mathrm{w} / \mathrm{v})$ in ethanol in a sealed glass bottle with magnetic stirring at $700 \mathrm{rpm}$, and distilled water was added slowly at a constant rate of $200 \mu \mathrm{L} \cdot \mathrm{min}^{-1}$ at room temperature. After the addition of water, stirring was continued for an additional $5 \mathrm{~min}$. The mixed solution was placed in an ultrasonic apparatus in an ice bath for predetermined time intervals ( $150 \mathrm{~W}, 5 \mathrm{~s}$ and $3 \mathrm{~s}$ apart). The ethosomes were filtered through a $0.22 \mu \mathrm{m}$ microporous membrane filter, protected from light, and then stored at $4^{\circ} \mathrm{C}$ until use. Blank control ethosomes were prepared using the same procedure, but without Rhodamine B.

Liposomes were prepared using the thin layer evaporation technique, ${ }^{24}$ in which the contents of yolk phospholipids and Rhodamine B were identical to the optimized ethosome formulation. Briefly, a chloroform solution of the required lipid mixture was first dried under vacuum overnight, and the resultant lipid film was hydrated with distilled water to prepare the lipid suspension. The suspension was then sonicated for the same time as the optimized ethosomal 
preparation, protected from light, and stored at $4^{\circ} \mathrm{C}$ until use. For preparation of the Rhodamine B hydroethanolic solution, the contents of ethanol and Rhodamine B were identical to those in the optimized ethosomal preparation.

\section{Separation of nonentrapped Rhodamine B from ethosomes}

Rhodamine B-loaded, NBD-PC-labeled ethosomes (phospholipid:NBD-PC $=100$ mole: 1 mole) were prepared using the aforementioned methods. ${ }^{25}$ Nonentrapped Rhodamine B was separated from the NBD-PC-labeled ethosomes by centrifugation (Thermo Scientific Sorvall Legend Mach; Thermo Fisher Scientific, Waltham, MA, USA). In brief, the ethosomes were added to an ultrafiltration tube (EMD Millipore, Billerica, MA, USA), with a cut-off molecular weight of 3,000 Da, and centrifuged at $11,185 \mathrm{rpm}$ and $4^{\circ} \mathrm{C}$ for $60 \mathrm{~min}$. The filtrate was removed, and the retentate tube was then inverted into a new collection tube to collect the entrapped ethosomes, which was centrifuged at 2,990 rpm and $4{ }^{\circ} \mathrm{C}$ for $5 \mathrm{~min}$. The entrapped Rhodamine B-loaded, NBD-PC-labeled ethosomes were used immediately in the subsequent skin penetration experiments.

\section{Characterization of the ethosomes}

The vesicle size and polydispersity index of Rhodamine B-loaded ethosomes and liposomes were determined using a dynamic light scattering method, with a Zetasizer Nano ZS90 (Malvern Instruments Ltd., Malvern, UK) without dilution or concentration.

\section{In vivo skin penetration study in rats}

The time-dependent permeation behavior of Rhodamine $B$ from the ethosomal system in rats ( $n=6$ per group) was measured by confocal laser scanning microscopy (CLSM) $)^{26}$ and compared with two control systems: liposomes and a hydroethanolic solution. A mini-funnel with a diameter of $3 \mathrm{~cm}$, which served as a drug pool, was stuck with glue to the rat abdominal skin that had been shaved carefully. Different formulations $(200 \mu \mathrm{L})$ were added into the funnels. Three rats from each group were selected randomly, and the treated skin samples were removed at 1,4 , and $8 \mathrm{~h}$ after test compound application. The experiments for the other three rats in each group lasted for $8 \mathrm{~h}$, and then the test compound was removed. The application areas were cleaned, and the treated skin samples were collected at 1, 4, and $8 \mathrm{~h}$. The skin tissue samples were embedded in embedding medium, sectioned at $8 \mu \mathrm{m}$ thicknesses immediately, with a cryo-ultramicrotome, and observed with CLSM, with an excitation wavelength of
$556 \mathrm{~nm}$. The blank group ethosomes were used as a control. All procedures were carried out in the dark to prevent the influence of ambient light. The fluorescence intensity of each image was measured by Image-Pro Plus software (Image-Pro Plus 6.0; Media Cybernetics, Washington DC, USA).

\section{Penetration mechanism study in rats}

Sprague Dawley rats were randomly divided into three groups ( $\mathrm{n}=5$ per group) for different treatment durations: 1,4 , and $8 \mathrm{~h}$. Rhodamine B-loaded, NBD-labeled ethosomes were administered as above, and the skin tissue samples were collected at 1,4 , and $8 \mathrm{~h}$ after drug application, and processed as above for observation by CLSM. In this study, the two probes used could be visualized with different excitations. A helium-neon laser (excitation wavelength: $558 \mathrm{~nm}$ ) was applied to visualize the vesicle and Rhodamine $B$ distribution in the treated skin samples (red), and an argon laser (excitation wavelength: $478 \mathrm{~nm}$ ) was applied to visualize NBD-PC in the ethosomes (green).

\section{Statistical analysis}

All quantitative data are expressed as the mean \pm standard deviation of three measurements. Statistical analyses were accomplished by analysis of variance or the Student's $t$-test, using SPSS software (SPSS Statistics 20.0; IBM Corporation, Armonk, NY, USA). A value of $P<0.05$ was considered statistically significant.

\section{Results \\ Ethosomal optimization and characteristics of the vesicles}

To determine the optimal formulation for the ethosomes, a total of 17 runs of the Box-Behnken experimental design were performed. The results of the responses (vesicle size) are shown in Table 1, and the three-dimensional plots are shown in Figure 1. All three independent variables, including yolk phospholipid content, ethanol content, and ultrasonic time, had interactive effects on the vesicle size of the ethosomes. The optimal design was determined to be $2.45 \%(\mathrm{w} / \mathrm{v})$ yolk phospholipids, 30\% (v/v) ethanol, and an ultrasonic time of $8 \mathrm{~min}$. The predicted value of the vesicle size was $132.4 \mathrm{~nm}$, and the measured mean value was $125.7 \mathrm{~nm}$.

The vesicle size and polydispersity index of the Rhodamine B-loaded ethosome vesicles were determined by dynamic light scattering. The mean vesicle size of the ethosomes was $125.7 \pm 3.2 \mathrm{~nm}(\mathrm{n}=3)$, while the mean polydispersity index was $0.199 \pm 0.014(n=3)$. The mean size of the liposomes was $185.9 \pm 1.8 \mathrm{~nm}(\mathrm{n}=3)$, while the mean polydispersity index was $0.289 \pm 0.012(\mathrm{n}=3)$. 
Table I Box-Behnken study design and experimental results

\begin{tabular}{lllll}
\hline Run & $\begin{array}{l}\text { Yolk phospholipid } \\
(\mathbf{w} / \mathbf{v})\end{array}$ & $\begin{array}{l}\text { Ethanol } \\
(\mathbf{v} / \mathbf{v})\end{array}$ & $\begin{array}{l}\text { Ultrasonic } \\
\text { time (min) }\end{array}$ & $\begin{array}{l}\text { Particle } \\
\text { size (nm) }\end{array}$ \\
\hline 1 & 0 & 1 & -1 & 182.8 \\
2 & -1 & 1 & 0 & 176.5 \\
3 & 0 & 0 & 0 & 187.2 \\
4 & 0 & 0 & 0 & 188.7 \\
5 & -1 & 0 & -1 & 178.9 \\
6 & 1 & 1 & 0 & 197.2 \\
7 & 1 & 0 & 1 & 213.4 \\
8 & 0 & -1 & -1 & 198.1 \\
9 & 0 & 0 & 0 & 183.7 \\
10 & 0 & 0 & 0 & 189 \\
11 & 0 & 1 & 1 & 123.5 \\
12 & 0 & -1 & 1 & 195.2 \\
13 & -1 & -1 & 0 & 188.2 \\
14 & 1 & -1 & 0 & 225.4 \\
15 & -1 & 0 & 1 & 175.3 \\
16 & 1 & 0 & -1 & 236.3 \\
17 & 0 & 0 & 0 & 183.4 \\
\hline
\end{tabular}

\section{Skin penetration properties}

The quantitative data from the experiments are shown in Table 2 and Figure 2. Fluorescent photomicrographs of the skin treated with Rhodamine B-labeled ethosomes, liposomes, and 30\% hydroethanolic solution are shown
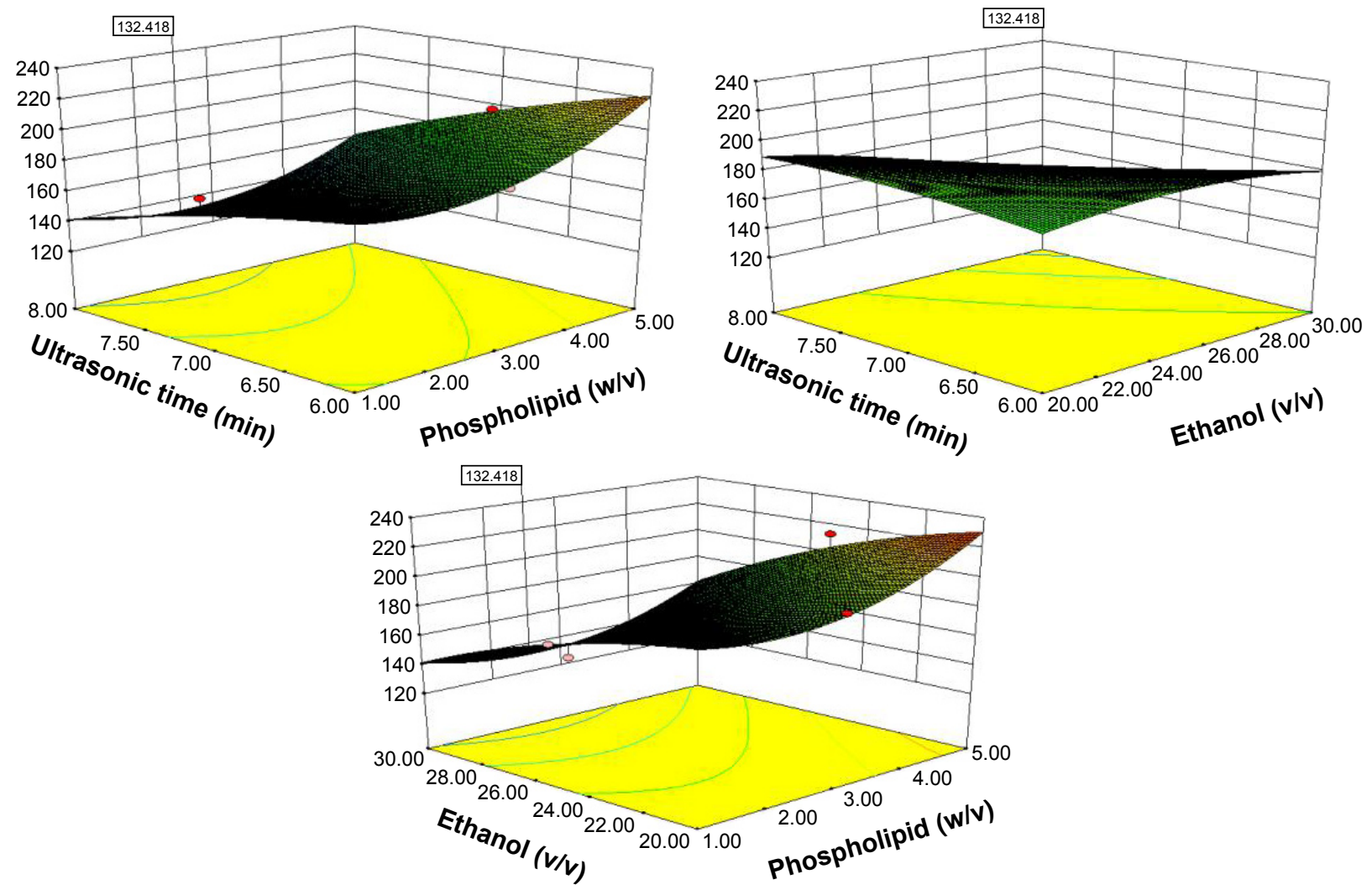

Figure I Three-dimensional response surface plots showing the effects of the independent variables (phospholipid, ethanol, and ultrasonic time) on the vesicle size of ethosomes.

in Figure 3. Enhanced delivery of Rhodamine B in terms of depth (viable epidermis, dermis, hair follicle, and even adipose tissue) was observed when it was loaded in ethosomes. In contrast, the penetration of Rhodamine B from conventional liposomes was limited to the upper layer of the skin (stratum corneum), with slight or negligible penetration observed in the viable epidermis and dermis (Figure 3C). Furthermore, the presence of fluorescence in the skin appendages pathway indicated that Rhodamine B-labeled ethosomes penetrated the skin via this pathway. A 30\% hydroethanolic solution of Rhodamine B (Figure 3B) showed much weaker fluorescence in comparison with the ethosomal formulation (Figure 3A), but it displayed a stronger fluorescence than liposomes (Figure 3C). The control ethosome samples did not show any fluorescence (data not shown).

\section{Mechanistic observation of skin penetration}

CLSM with double fluorescence-labeled ethosomes illustrated the permeation process of ethosomes loaded with lipophilic compounds into the deep layers of the skin (Figure 4). The presence of red and green fluorescence in the skin tissues indicated that both Rhodamine B and NBD-PC 
Table 2 The mean fluorescence intensity (AU, mean \pm standard deviation, $n=3$ ) of tissue slices of Rhodamine B-loaded ethosomes, liposomes, and hydroethanolic solution at I, 4 , and $8 \mathrm{~h}$ after test compound administration and $\mathrm{I}, 4$, and $8 \mathrm{~h}$ after removal of the test compounds from the skin

\begin{tabular}{|c|c|c|c|c|c|c|}
\hline \multirow[t]{3}{*}{ Formulation } & \multicolumn{6}{|l|}{ Time (h) } \\
\hline & \multicolumn{3}{|c|}{ Time after transdermal test compound delivery } & \multicolumn{3}{|c|}{ Time after test compound removal from the skin } \\
\hline & I h & $4 \mathrm{~h}$ & $8 \mathrm{~h}$ & I h & $4 \mathrm{~h}$ & $8 \mathrm{~h}$ \\
\hline Ethosomes & $57.479 \pm 7.331$ & $68.08 \pm 3.158$ & $87.997 \pm 3.985$ & $55.524 \pm 1.586$ & $31.065 \pm 1.626$ & $23.777 \pm 2.052$ \\
\hline Liposomes & $20.207 \pm 0.564$ & $25.435 \pm 0.573$ & $28.55 I \pm 1.276$ & $25.258 \pm 0.125$ & $15.982 \pm 0.523$ & $9.24 \pm 0.900$ \\
\hline $\begin{array}{l}\text { Hydroethanolic } \\
\text { solution }\end{array}$ & $22.808 \pm 0.725$ & $28.399 \pm 1.028$ & $35.412 \pm 0.696$ & $30.005 \pm 0.485$ & $20.05 I \pm 0.499$ & $14.413 \pm 0.543$ \\
\hline
\end{tabular}

could penetrate through the skin. If their intensities appeared differently or only one fluorescent color existed in the deep layers, it would indicate that the vesicles were broken and that the entrapped drug was released into the tissues alone. At $1 \mathrm{~h}$ after test compound administration, the red and green fluorescence exhibited a slight, continuous strip distribution, which was consistent in the stratum corneum and viable epidermis. In hair follicles of the superficial layer, the red fluorescence was distributed in the perifollicular epithelial tissues and connective tissues, while the green fluorescence was distributed in the hair root and hair shaft; separation of fluorescent colors was observed in the hair follicle, but not in the stratum corneum. Four hours later, the fluorescence from both Rhodamine B and NBD-PC became brighter: bright red fluorescence became visible in the deep layer of the dermis and hair follicles and green fluorescence remained in the viable epidermis, indicating that the distribution difference between the two fluorescent compounds emerged. At $8 \mathrm{~h}$, a broader distribution of red fluorescence without green fluorescence was seen in the dermis or deep layer of the skin (adipose tissue). In addition, the distribution of Rhodamine B and NBD-PC appeared obviously different. Moreover, the distribution pattern of ethosomes was different from that of liposomes because the liposomes were confined in the upper layers of the stratum corneum and even prevented the test compounds from passing through the deeper layer.

\section{Discussion}

Many factors influence the delivery of drugs into the skin from topically applied vesicles; the vesicle size and encapsulation efficiency are the key parameters. ${ }^{27}$ Smaller vesicles
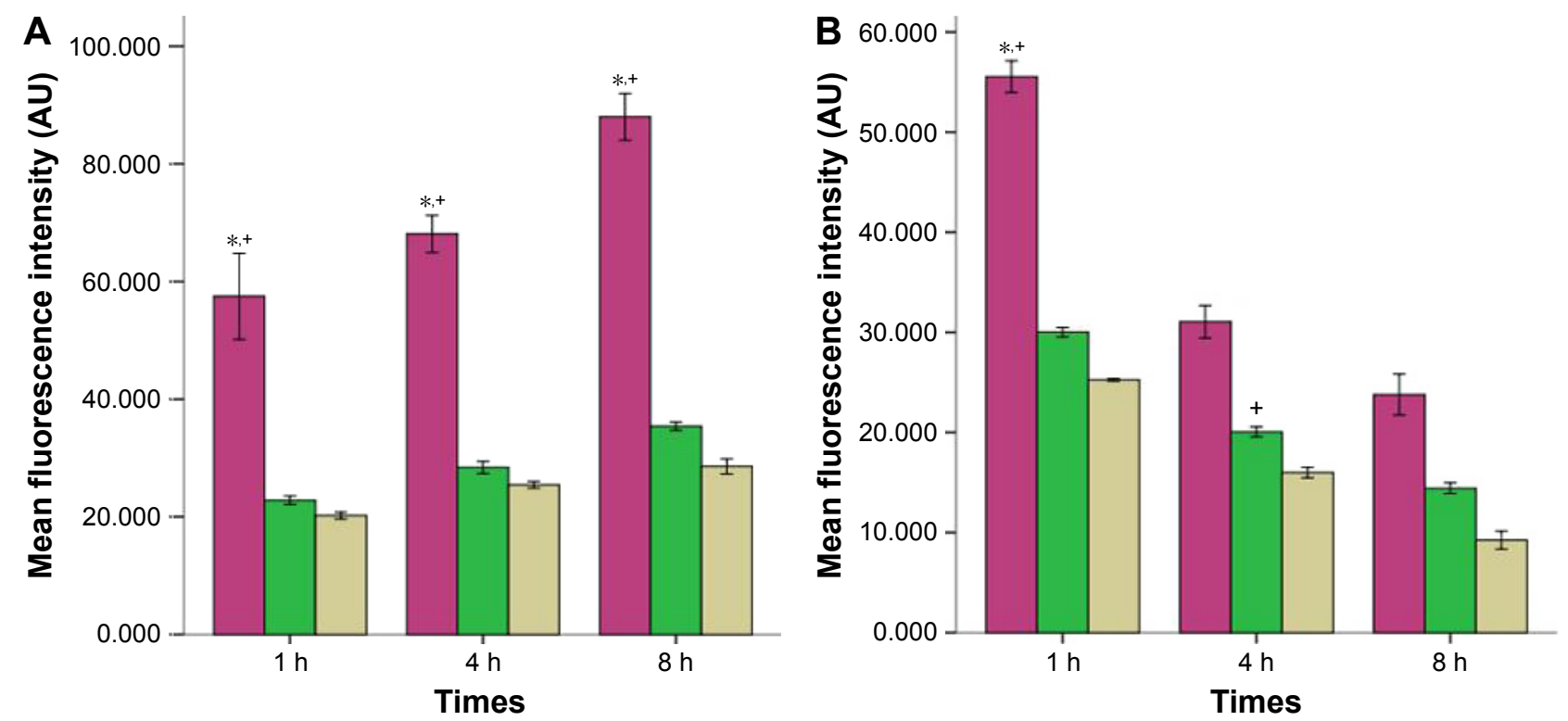

Ethosomes Hydroethanolic solution Liposomes

Figure 2 Mean fluorescence intensity of Rhodamine B-loaded ethosomes, hydroethanolic solution, and liposomes at I, 4, and $8 \mathrm{~h}$ after application (A) as well as I, 4, and $8 \mathrm{~h}$ after the test compound was removed from the skin (B). ${ }^{* P}<0.05$ for the comparison with hydroethanolic solution, ${ }^{+} P<0.05$ for the comparison with liposomes. 

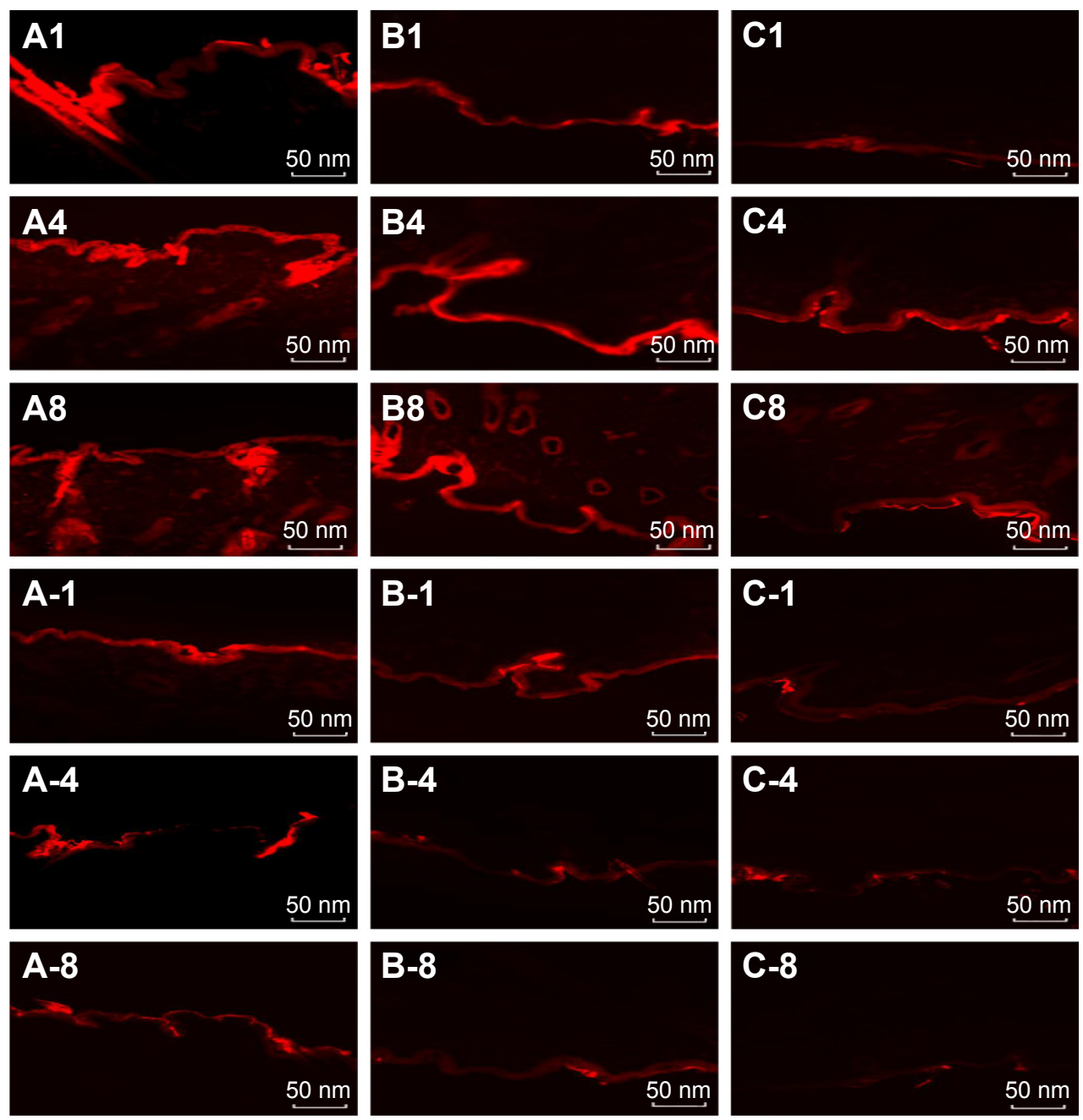

Figure 3 Confocal laser scanning microscopy images of rat skin samples from the skin penetration study of Rhodamine B-loaded ethosomes (A), hydroethanolic solution (B), and liposomes (C) at I, 4, and $8 \mathrm{~h}$ after application (AI, A4, A8, BI, B4, B8, CI, C4, and C8) as well as I, 4, and $8 \mathrm{~h}$ after the test compound was removed from the skin (A-I, A-4, A-8, B-I, B-4, B-8, C-I, C-4, and C-8).

are able to deliver their encapsulated drugs into the deeper layers of the skin, ${ }^{28}$ and like other studies, the vesicle size was chosen as an index for efficiency and feasibility in our experiments. Our data (Figure 1) show that the vesicle size of the ethosomes decreased as the ethanol concentration increased, but it increased as the phospholipid concentration increased, indicating that the factors, in descending order, that could influence the vesicle size were the yolk phospholipid content, ethanol content, and ultrasonic treatment time.

Our previous study ${ }^{15}$ and subsequent other studies have proven that ethosomes can improve percutaneous permeation of drugs. ${ }^{29-31}$ In terms of possible mechanisms for ethosomes to promote percutaneous permeation of drugs, two viewpoints have been presented: the first one is that ethanol is a known penetration enhancer, which can disturb the multilamellar and ordered lipid domain, lower the structure density, and enhance the fluidity of the stratum corneum, thus providing the vesicles with flexible characteristics that allow them to penetrate easily into deeper layers of the skin; ${ }^{32,33}$ the second one is that phospholipids between the stratum corneum and ethosomal vesicle fuse easily, thus changing the transition temperature, leading to enhanced drug penetration. ${ }^{34}$ In our research, we interestingly found that even though the lipid content in the liposomes and the ethanol content in the hydroethanolic solution were equal with those in the ethosomes, after percutaneous penetration, a greater fluorescence intensity in the skin of the ethosomal system was observed, compared with that in the hydroethanolic solution and liposomes $(P<0.05$; Figure 2B). These results further confirmed that alcohol or phospholipid content was not the only driving force for promoting effective drug penetration into the skin; therefore, we speculate that there is a synergistic mechanism among lipids, ethanol, and the skin, by which skin penetration of drugs can be obviously promoted. Also, ethosomes can 

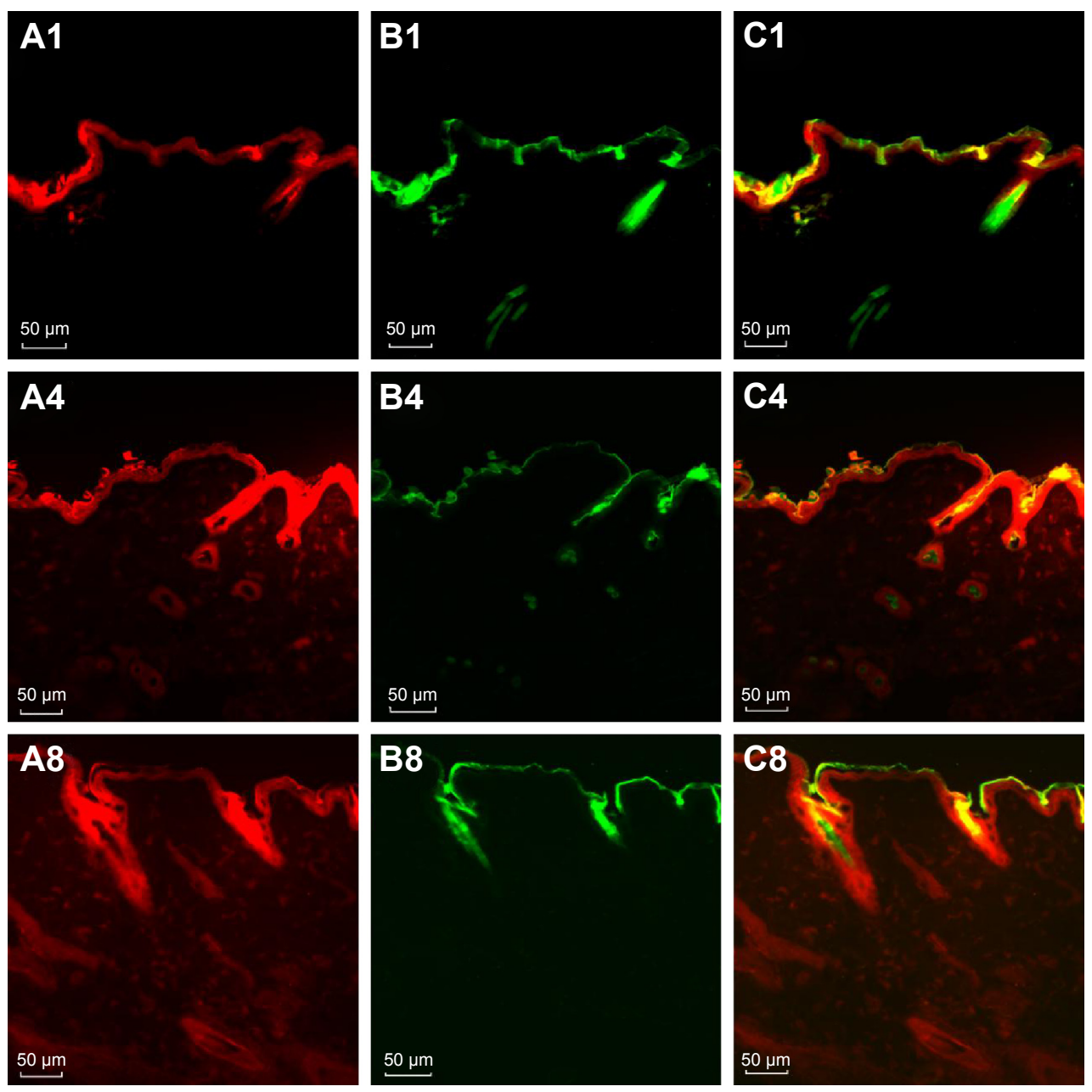

Figure 4 Confocal laser scanning microscopy images of rat samples from the skin penetration study of Rhodamine B-loaded, NBD-PC-labeled ethosomes at I, 4, and $8 \mathrm{~h}$ after application (AI, A4, A8, B I, B4, B8, CI, C4, and C8).

Notes: (A) Red fluorescence of Rhodamine B; (B) green fluorescence of NBD-PC; (C) overlay of A and B.

Abbreviation: NBD-PC, I-palmitoyl-2-\{I2-[(7-nitro-2-I,3-benzoxadiazol-4-yl)amino]dodecanoyl\}-sn-glycero-3-phosphocholine.

penetrate into the skin via skin appendages; the hair follicle and stratum corneum have a remarkably longer retention time compared with the other two preparations. Nevertheless, we did not find the existence of green fluorescence-labeled phospholipids in the dermis. This difference reminded us that ethosomes experience sphere separation and drug release in the superficial epidermis.

Compared with liposomes and the hydroethanolic solution, ethosomes have a particularly longer duration in skin appendages and the dermis, suggesting that ethosomes are especially suitable to be the carrier of some drugs through the skin, such as the drugs for hair follicle and sebaceous gland diseases, photodynamic therapy, and anesthetics.

\section{Conclusion}

Based on our results from this study, we conclude that ethosomes as a percutaneous drug carrier showed transdermal superiority over liposomes and hydroethanolic solution, due to the synergistic effect of their ingredients with the skin structures. Of note, during the percutaneous process, the vesicles might break up in the superficial layer of skin, allowing drugs to permeate into the deeper layer alone, thus allowing the phospholipid to be retained in the upper epidermis.

\section{Acknowledgments}

We are grateful to all of our colleagues working in the Department of Dermatology, Nanfang Hospital, Southern Medical University for their help. We would also like to acknowledge the useful comments on this paper that were received from reviewers. This work was supported by the Natural Science Foundation of Guangdong Province, China (Grant number 2014A030313320), the Medical Scientific Research Foundation of Guangdong Province, China (Grant number A2016544), Science and Technology Development 
Program of Southern Medical University (C1033041), and the Science and Technology Planning Project of Guangzhou, China (Grant number 201510010120).

\section{Disclosure}

The authors report no conflicts of interest related to this work.

\section{References}

1. Ben MM, Khalfallah M, Boutiba Ben Boubaker I, et al. Bacteriological and therapeutic profile of diabetic foot infection: a prospective study of 100 patients. Tunis Med. 2016;94(2):95-101.

2. Kim SY, Lee YW, Choe YB, Ahn KJ. Progress in Malassezia Research in Korea. Ann Dermatol. 2015;27(6):647-657.

3. Haspeslagh M, Taevernier L, Maes AA, et al. Topical distribution of acyclovir in normal equine skin and equine sarcoids: an in vitro study. Res Vet Sci. 2016;106:107-111.

4. Lam LH, Sugarman JL. Adrenal suppression with chronic topical corticosteroid use in psoriasis patients. J Drugs Dermatol. 2016;15(8): 945-948.

5. Korobko IV, Lomonosov KM. A pilot comparative study of topical latanoprost and tacrolimus in combination with narrow-band ultraviolet B phototherapy and microneedling for the treatment of nonsegmental vitiligo. Dermatol Ther. 2016;29(6):437-441.

6. Kushwaha A, Shivakumar HN, Murthy SN. Iontophoresis for drug delivery into the nail apparatus: exploring hyponychium as the site of delivery. Drug Dev Ind Pharm. 2016;42(10):1678-1682.

7. Petchsangsai M, Rojanarata T, Opanasopit $\mathrm{P}$, Ngawhirunpat T. The combination of microneedles with electroporation and sonophoresis to enhance hydrophilic macromolecule skin penetration. Biol Pharm Bull. 2014;37(8):1373-1382.

8. Larraneta E, Lutton RE, Brady AJ, et al. Microwave-assisted preparation of hydrogel-forming microneedle arrays for transdermal drug delivery applications. Macromol Mater Eng. 2015;300(6):586-595.

9. Tokumoto S, Higo N, Todo H, Sugibayashi K. Effect of combination of low-frequency sonophoresis or electroporation with iontophoresis on the mannitol flux or electroosmosis through excised skin. Biol Pharm Bull. 2016;39(7):1206-1210.

10. Tamakuwala M, Stagni G. Fingolimod hydrochloride gel for dermatological applications: optimization of formulation strength and effect of colloidal oatmeal (Aveeno $\left.{ }^{\circledR}\right)$ as penetration enhancer. AAPS PharmSciTech. 2016;17(4):907-914.

11. Liu X, Li Z, Wang X, et al. Novel antimicrobial peptide\&ndash; modified azithromycin-loaded liposomes against methicillin-resistant Staphylococcus aureus. Int J Nanomedicine. 2016;11:6781-6794.

12. Ita K. Current status of ethosomes and elastic liposomes in dermal and transdermal drug delivery. Curr Pharm Des. 2016;22(33): $5120-5126$.

13. Akhtar N, Verma A, Pathak K. Ethosomes as vesicles for effective transdermal delivery: from bench to clinical implementation. Curr Clin Pharmacol. 2016;11(3):168-190.

14. Iizhar SA, Syed IA, Satar R, Ansari SA. In vitro assessment of pharmaceutical potential of ethosomes entrapped with terbinafine hydrochloride. J Adv Res. 2016;7(3):453-461.

International Journal of Nanomedicine

\section{Publish your work in this journal}

The International Journal of Nanomedicine is an international, peerreviewed journal focusing on the application of nanotechnology in diagnostics, therapeutics, and drug delivery systems throughout the biomedical field. This journal is indexed on PubMed Central, MedLine, CAS, SciSearch $\AA$, Current Contents ${ }^{\circledR} /$ Clinical Medicine,
15. Zhu X, Li F, Peng X, Zeng K. Formulation and evaluation of lidocaine base ethosomes for transdermal delivery. Anesth Analg. 2013; 117(2):352-357.

16. Rkein AM, Ozog DM. Photodynamic Therapy. Dermatol Clin. 2014; 32(3):415-425.

17. Abrahamse H, Hamblin MR. New photosensitizers for photodynamic therapy. Biochem J. 2016;473(4):347-364.

18. Ganesan P, Choi DK. Current application of phytocompound-based nanocosmeceuticals for beauty and skin therapy. Int $J$ Nanomedicine. 2016;11:1987-2007.

19. Jain S, Tiwary AK, Sapra B, Jain NK. Formulation and evaluation of ethosomes for transdermal delivery of lamivudine. AAPS PharmSciTech. 2007;8(4):E111.

20. Yu Z, Lv H, Han G, Ma K. Ethosomes loaded with cryptotanshinone for acne treatment through topical gel formulation. PLoS One. 2016;11(7):e159967.

21. Iizhar SA, Syed IA, Satar R, Ansari SA. In vitro assessment of pharmaceutical potential of ethosomes entrapped with terbinafine hydrochloride. J Adv Res. 2016;7(3):453-461.

22. Gannu R, Palem CR, Yamsani SK, Yamsani VV, Yamsani MR. Enhanced bioavailability of buspirone from reservoir-based transdermal therapeutic system, optimization of formulation employing box - behnken statistical design. AAPS PharmSciTech. 2010;11(2):976-985.

23. Touitou E, Dayan N, Bergelson L, Godin B, Eliaz M. Ethosomes novel vesicular carriers for enhanced delivery: characterization and skin penetration properties. J Control Release. 2000;65(3):403-418.

24. Rakesh R, Anoop KR. Formulation and optimization of nano-sized ethosomes for enhanced transdermal delivery of cromolyn sodium. J Pharm Bioallied Sci. 2012;4(4):333-340.

25. Dadashvand N, Williams LA, Othon CM. Heterogeneous rotational diffusion of a fluorescent probe in lipid monolayers. Struct Dyn. 2014; 1(5):054701.

26. Alvarez-Román R, Naik A, Kalia YN, Fessi H, Guy RH. Visualization of skin penetration using confocal laser scanning microscopy. Eur $J$ Pharm Biopharm. 2004;58(2):301-316.

27. Choi JH, Cho SH, Yun JJ, Yu YB, Cho CW. Ethosomes and transfersomes for topical delivery of ginsenoside $\mathrm{Rhl}$ from red ginseng: characterization and in vitro evaluation. J Nanosci Nanotechnol. 2015;15(8):5660-5662.

28. Verma DD, Verma S, Blume G, Fahr A. Particle size of liposomes influences dermal delivery of substances into skin. Int J Pharm. 2003; 258(1-2):141-151.

29. Marto J, Vitor C, Guerreiro A, et al. Ethosomes for enhanced skin delivery of griseofulvin. Colloids Surf B Biointerfaces. 2016;146:616-623.

30. Akhtar N, Verma A, Pathak K. Ethosomes as vesicles for effective transdermal delivery: from bench to clinical implementation. Curr Clin Pharmacol. 2016;11(3):168-190.

31. Yu Z, Lv H, Han G, Ma K. Ethosomes loaded with cryptotanshinone for acne treatment through topical gel formulation. PLoS One. 2016;11(7):e159967.

32. Bodade SS, Shaikh KS, Kamble MS, Chaudhari PD. A study on ethosomes as mode for transdermal delivery of an antidiabetic drug. Drug Deliv. 2013;20(1):40-46.

33. Touitou E, Godin B. Ethosomes for skin delivery. J Drug Deliv Sci Technol. 2007;17(5)303-308.

34. Yu X, Du L, Li Y, Fu G, Jin Y. Improved anti-melanoma effect of a transdermal mitoxantrone ethosome gel. Biomed Pharmacother. 2015;73:6-11.

Journal Citation Reports/Science Edition, EMBase, Scopus and the Elsevier Bibliographic databases. The manuscript management system is completely online and includes a very quick and fair peer-review system, which is all easy to use. Visit http://www.dovepress.com/ testimonials.php to read real quotes from published authors. 